\title{
Structural Models of Amorphous Carbon and its Surfaces by Tight-Binding Molecular Dynamics
}

R. Haerle, A. Baldereschi and G. Galli

This article was submitted to $18^{\text {th }}$ International Conference on Amorphous and Microcrystalline Semiconductors

Snowbird, UT

August 22-27, 1999

U.S. Department of Energy

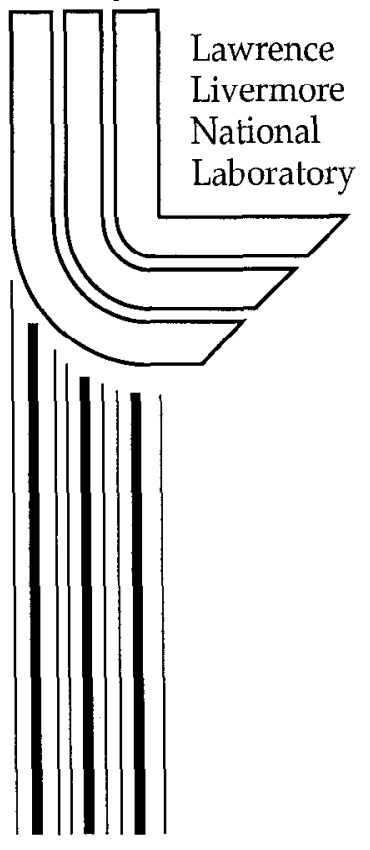

October 26, 1999 


\section{DISCLAIMER}

This document was prepared as an account of work sponsored by an agency of the United States Government. Neither the United States Government nor the University of California nor any of their employees, makes any warranty, express or implied, or assumes any legal liability or responsibility for the accuracy, completeness, or usefulness of any information, apparatus, product, or process disclosed, or represents that its use would not infringe privately owned rights. Reference herein to any specific commercial product, process, or service by trade name, trademark, manufacturer, or otherwise, does not necessarily constitute or imply its endorsement, recommendation, or favoring by the United States Government or the University of California. The views and opinions of authors expressed herein do not necessarily state or reflect those of the United States Government or the University of California, and shall not be used for advertising or product endorsement purposes.

This is a preprint of a paper intended for publication in a journal or proceedings. Since changes may be made before publication, this preprint is made available with the understanding that it will not be cited or reproduced without the permission of the author.

This report has been reproduced directly from the best available copy.

Available to DOE and DOE contractors from the Office of Scientific and Technical Information

P.O. Box 62, Oak Ridge, TN 37831

Prices available from (423) 576-8401 http:/ /apollo.osti.gov/bridge/

Available to the public from the National Technical Information Service

U.S. Department of Commerce 5285 Port Royal Rd., Springfield, VA 22161 http://www.ntis.gov/

$$
\text { OR }
$$

Lawrence Livermore National Laboratory Technical Information Department's Digital Library http:/ / www.llnl.gov/tid/Library.html 


\title{
Structural Models of Amorphous Carbon and its Surfaces by Tight-Binding Molecular Dynamics
}

\author{
Rainer Haerle ${ }^{(a)}$, Alfonso Baldereschi ${ }^{(a)}$, Giulia Galli ${ }^{(b)}$ \\ (a) Institute of Applied Physics, EPF-Lausanne and IRRMA, 1015 Lausanne, Switzerland \\ (b) Lawrence Livermore National Laboratory, CA, USA
}

We use liner-scaling tight-binding molecular dynamics to generate three structural models of bulk amorphous carbon with different atomic density. Amorphous carbon surfaces are then obtained by imposing tensile strain on these computer generated networks until fracture occurs. Our results show that for a given density, the formation energy of surfaces obtained with different tensile strains differ by only a few $10^{-1} \mathrm{eV} /$ atom and their structural properties are qualitatively similar. The prescnce of $s p$ sitcs at the surface is observed at all densities, but with different values of the concentration. The surface thickncsses obtained in our simulations agree with experimental data. Furthermore we find that surface roughness increascs with the amount of graphitic component in the bulk sample. The samc trends of the macroscopic properties are obtained when using a two-center tight-binding Hamiltonian, an environmental dependent one, and first principles calculations.

\section{INTRODUCTION}

Many experimental [1,2] and theoretical [3-5] investigations have been performed so far in order to understand the properties of bulk amorphous carbon (a-C), a disordered phase of carbon without long range order and containing carbon atoms in different hybridization slates. Depending on the preparation technique [6], phases with various macroscopic properties can be produced; notably the atomic density and the ratio of $s p^{2} / s p^{3}$ hybridization can vary significantly from sample to sample.

On the contrary, only a limited number of studies have dealt so far with the characterisa- 
tion of the surface properties of this technological important material. Surface investigations are most relevant for many applications of a-C, ranging from wear resistant coatings for mechanical and biological applications [7], to potential cold emitters for flat panel displays [8].

Experimentally, the a-C surface roughness has been studied using atomic force microscopy (AFM) [9-11], and the $s p^{3}$ site-density at the surface has been obtained with spatially resolved cross sectional EELS measurements [12]. Theoretical investigations are based on a variety of computational molecular-dynamics schemes, including first-principles and tight-binding Hamiltonians, as well as empirical potentials, and focus mainly on the generation of bulk a-C networks. To the best of our knowledge, only three studies of a-C surfaces have appeared in the literature so far [13-15]. The first study on a-C was done with an empirical Tersoff potential [13] by heating a carbon slap, another is a semiempirical study of an a-C computer-generated network, arbitrarily terminated in one direction with a sharp cut, thus generating a surface [14]; the third reports about simulations of ion subplantation during the growth process of diamond-like carbon [15].

In this paper we present the results of a series of molecular dynamics (MD) simulations, done in a two-center tight-binding (TC-TB) [16] framework, aimed at modelling a-C surfaces of samples with various macroscopic atomic densities. These results are then compared with those obtained using an enviromental-dependent three-center tight-binding (ED-TB) scheme, and, for the case of a small network of $112 \mathrm{C}$ atoms, are also checked against the results of self-consistent first-principles calculations. The results of all these calculations provide the same trends for the macroscopic structural properties of a-C surfaces and they comparc well with availablc cxpcrimental data.

\section{METHODS}

The MD simulations have been performed with tight-binding (TB) Hamiltonians since they provide the simplest quantum mechanical description of chemical bonds and give direct. 
access to electronic properties, contrary to investigations based on interatomic potentials. At each time step of the MD simulation, the electronic problem has been solved quantum mechanically using either the TC-TB Hamiltonian proposed in Ref. [16] or the environmentaldependent (ED-TB) three-center one, proposed in Ref. [17]. Both hamiltonians have been obtained by fitting matrix elements to first principles calculations and are based on a simple, orthogonal, minimal basis set. In all calculations we have used a linear scaling method [18] so that we can handle relatively large systems (our periodically repeated supercells contain 512 carbon atoms) for total simulation times as long as 120 ps.

In our TC-TB simulations, we first generated bulk networks with varying $s p^{2} / s p^{3}$ ratio, in order to investigate how different preparation conditions influence the surface structure. Three different a-C networks were obtained by an annealing and quenching technique, starting from molten samples of $512 \mathrm{C}$ atoms and quenching them to a disordered solid phase in about 10 ps. The resulting bulk a-C networks have different atomic density $(\rho=2.2,3.0$ and $4.0 \mathrm{~g} \cdot \mathrm{cm}^{-3}$ ), and 3 -fold/4-fold coordinated percentages of 77/14, 56/42 and 20/80 respectively; coordination numbers were determined using a cutoff distance of $1.8 \AA$ to define nearest neighbours, a value which lays well within the first dip of the pair correlation function for all three systems. We label the networks as $\rho 2-\mathrm{TC}, \rho 3-\mathrm{TC}$ and $\rho 4-\mathrm{TC}$, according to the value of the atomic density.

The a-C surfaces are then obtained by simulating the application of tensile strain on the computer-generated bulk networks until fracture occurs. Strain has been applied by progressively changing the dimension of one axis of the MD simulation cell and different preparation conditions have bcen mimicked with different strain simulation schemes. The total strain was applied in a scquencc of 15 to 30 discretc stcps, and aftcr cach stcp the system was allowed to relax to the closest ionic configuration of minimum energy. We used a displacement step $t=0.5 \AA$ and simulation times of 7 to $9 \mathrm{ps}$ wcre typically required to produce the fracture of the bulk network. Fig. 1 shows the microfracture process for a typical application of tensile strain on such a network. Four selected snapshots are presented in the figure, more examples with different displacement steps can be found in [19], together 
with a surface network, obtained by producing a surface via a sharp cut of the bulk network $\rho 3-\mathrm{TC}$.

Although the TC-TB hamiltonian has proved to be efficient and qualitatively accurate in several instances [5], it does not describe properly some important features of the local structure: for example, it underestimates the $s p^{3} / s p^{2}$ fraction in a-C systems with densities in the range 3.0 to $4.0 \mathrm{~g} \cdot \mathrm{cm}^{-3}$, furthermore, no 3-fold rings and only a minimal amount of 4-fold rings are present in the TC-TB a-C networks. The existence of 3 - and 4-fold rings has been well established in other works [4], and gives rise to caracteristic features: 3-fold rings have a very particular signature in vibrational spectra and 4-fold rings show up clearly in the pair-correlation function, as the C-C diagonal of the square $(\approx 2.1 \AA)$ causes a distinct peak. Deficiencies of the TC-TB scheme are not a major concern for us, since we focus more on the trends exhibited by the structural properties of surfaces, as a function of the bulk density, than on their absolute values. Nevertheless, we decided to check the quality of the TC-TB results by comparing them with those of the more sophisticated environmental-dependent ED-TB hamiltonian [17]. The latter is known to provide results similar to those of the TC-TB model, if a density rescaling of the sample is performed and limited to averaged macroscopic properties. In particular, the sarne $s p^{3} / s p^{2}$ ratio is oblained with the TC-TB and the ED-TB Hamiltonians, when considering a macroscopic density of $4.0 \mathrm{~g} \cdot \mathrm{cm}^{-3}$ in the former scheme and $3.0 \mathrm{~g} \cdot \mathrm{cm}^{-3}$ in the latter.

We produced two a-C networks with the ED-TB IIamiltonian with densities $\rho=3.0$ and $4.0 \mathrm{~g} \cdot \mathrm{cm}^{-3}$, by annealing and quenching them in the same way as for the TC-TB networks. We labeled these bulk networks $\rho 3$-ED and $\rho 4$-ED and calculated $s p^{3} / s p^{2}$ ratios of $65 / 33$ and 97/3. $\rho 3$-ED ( $\rho 4-\mathrm{ED})$ contains 4 (10) 3-fold and 23 (28) 4-fold rings.

The large supercells and long simulation times considered so far by us with TB hamiltonians are still out of reach of first-principles MD simulations. For cxample, the investigation of an a-SiC surface [20] using Density Functional Theory (DFT) in the Local Density Approximation (LDA) was limited to a MD cell with 128 atoms and a short simulation time. A DFT-LDA calculation on a small carbon system and for a limited simulation time, however, 
is feasible and we decided to investigate the soundness of the TC-TB and ED-TB results on two different, smaller a-C systems, containing 112 carbon atoms $\left(\rho=3.0 \mathrm{~g} \cdot \mathrm{cm}^{-3}\right)$ generated with the ED-TB and the TC-TB schemes by calculating for the resulting bulk networks and relaxed surfaces the forces with a micro-canonical first-principles DFT-LDA calculation [21]. We used a plane wave basis set with an energy cutoff of 35 RY for the expansion of the single particle orbitals, and norm-conserving pseudo-potentials. Only Bloch functions at the $\Gamma$-point of the Brillouin zone of the supercell have been taken into account [22]. Altogether four networks were examined with DFT: The TC-TB (ED-TB) bulk networks and the TCTB (ED-TB) surface networks, produced by the application of tensile strain as explained above. We started first with the TC-TB network and calculated its total energy and forces according to LDA. Then we allowed the atoms to relax to the closest local minimum of the LDA total energy. For convenience in comparing total energies, we used a reference cell big enough to accommodate also the two surfaces produced by tensile strain to define the basis set. Large atomic relaxations have been obtained in this case. The same procedure was repeated for the ED-TB bulk network and surfaces. No major structural change occurred and we observed only minor atomic relaxations. The surface formation energy, however, is reduced by the DFT-LDA relaxation $\left(0.13 \mathrm{eV} / \AA^{2}\right.$ instead of $0.16 \mathrm{eV} / \AA^{2}$ in the ED-TB case). This result confirmed the soundness of the ED-TB scheme and encouraged us to perform some of the calculations from the very beginning with the ED-TB IIamiltonian, by imposing tensile strains on $\rho 3$-ED and $\rho 4-\mathrm{ED}$.

\section{RESULTS}

We characterise the structural properties of the surfaces (two for each strain simulation), by calculating their formation energy $\Delta E$, thickness $\Delta z$, roughness $R_{a}$, number $N_{s}$ of atoms within a surface layer, and 3- and 4-fold coordination fractions $\left(N_{3} a n d N_{4}\right)$ within this layer. These results are summarised in Table $\mathrm{I}$. The average surface formation energy $\Delta E$ is computed as half the difference, per unit surface area, between the total energy of the system 
prior to and after fracture. We find that $\Delta E$ increases weakly for decreasing bulk density and does not vary appreciably as a function of strain conditions [19].

The surface formation energies observed with the ED model are much smaller than the ones steeming from the TC-TB Hamiltonian. This can be explained with the observation, that we calculated much larger values for the stress tensor in the ED case than in the 'I'C case. Since we kept the density fixed as an external constrain during the generation of the bulk, this stress energy diminishes the surface formation energy when the system is given room to expand (in one direction). Still, the observed structural trends are the same.

We define the surface thickness by considering the number of 1-, 2-, 3-, and 4-fold coordinated atoms within a $1 \AA$ wide layer parallel to the surface, as a function of the position of the central plane of the layer, along the direction perpendicular to the surface (compare Fig. 2). As the middle of the a-C network is approached, these quantities tend to their respective average values in the bulk before fracture. As the vacuum region between the two surfaces is approached, they drop to zero. These atomic densities as a function of the central plane position for the network $\rho 3-\mathrm{ED}$ are displayed in Fig. 2. We define the surface as the transient region of the slab where the atomic densities raise from zero to their bulk values. We counted the total number $N_{s}$ of atoms in the surface slab, whose thickness is $\Delta z$, and calculated the corresponding percentage of 3- and 4-fold coordinated atoms, reported as $N_{3}$ and $N_{4}$ in Table I. As a general trend, we find that the higher the density of the bulk (and correspondingly the fraction of $s p^{3}$ sites), the thinner the surface. Surface thicknesses of a-C samples with large $s p^{2} / s p^{3}$ ratio have been measured recently, and vary from $4 \pm 2$ to $13 \pm 3 \AA$, depending on the ion penetration depth (and thus the ion energy) during the film growth. Our results for the surface thickness of networks $\rho 3-\mathrm{TC}(3.5 \pm 1 \AA), \rho 4-\mathrm{TC}(1.7 \pm$ $0.3 \AA), \rho 3-\mathrm{ED}(3.2 \pm 0.4 \AA), \rho 4$-ED $(1.6 \pm 0.3 \AA)$ are consistent with these mcasurcments. We note that surfaces obtained for the $\rho 3$-TC nctwork with a sharp cut (see Rev. [19]) have smaller thickness, consistently with the results reported by Drabold et al. [12]. At all densities, the surface mostly contains 3-fold coordinated atoms, together with some 1-fold and some 2-fold coordinated ones. We obtained 2-fold coordinated atoms within the surface 
layer in all simulations. However, the ratio between the number of 3- and 2-fold coordinated surface atoms is significantly enhanced when using the ED-TB Hamiltonian, although the trend as a function of bulk density remains unchanged. Furthermore, average macroscopic properies such as surface roughness and thickness do not change much when going from one 'I'B scheme to the other, even if some features of the local structure are very different.

To make contact with AFM experiments on a-C films [9-11], we also calculated the surface roughness $R_{a}$ as $R_{a}=\sum \frac{\left|Z_{i}-Z_{a v e}\right|}{N}$ [11]. Experimentally, $N$ is the number of height measurements in AFM scans, $Z_{i}$ are the surface height values, and $Z_{\text {ave }}$ is the average height within a certain area. We assumed a tip size equal to the covalent radius of a carbon atom and we sampled the surface hight within the simulation cell using a $20 \times 20$ uniform grid. In agreement with the experimental data we find that the surface roughness increases significantly with decreasing density: a factor of 2 (3) in going from the bulk network $\rho 3$ $(\rho 4)$ to the network $\rho 2$ (for both, the TC and the ED model). A quantitative determination of surface roughness, however, has to be carried out using much bigger simulation cells.

\section{CONCLUSION}

In summary, we have studied structural models of computer generated a-C bulk networks and a-C surfaces and identified clear trends in their properties as a function of the macroscopic atomic density of the bulk. Our results are consistent with several experimental findings and indicate that surface properties depend weakly on the preparation conditions. The structural models generated in this work will be useful to evaluate electronic properties, and in particular to study the electron affinity of a-C surfaces, and to verify their claimed aptitude to cold emission.

Part of this work was performed by the Lawrence Livermore National Laboratory under the auspices of the U. S. Department of Energy, Office of Basic Energy Sciences, Division of Materials Science, Contract No. W-7405-ENG-48. 


\section{REFERENCES}

[1] P. .H. Gaskell, A. Saeed, P. Chieux, and D. R. McKenzie, Phys. Rev. Lett. 67, 1286 (1991)

[2] K. W. R. Gilkes,P. H. Gaskell, and J. Robertson, Phys. Rev. B 51, 12303 (1995)

[3] G. Galli, R. Martin, R. Car, and M. Parrinello, Phys. Rev. Lett. 62, 555 (1989); Phys. Rev. B 42, 7470 (1990)

[4] N. A. Marks, D. R McKenzie, B. A. Pailthorpe, M. Bernasconi, and M. Parrinello, Phys. Rev. Lett. 76, 768 (1996)

[5] C. Z. Wang, K. M. Ho, and C. T. Chan, Phys. Rev. Lett. 70, 611 (1993)

[6] See J. Robertson, Prog. Solid State Chem. 21, 199 (1991) for an overview.

[7] A. H. Lettington, in Diamond and Diamond-like Films and Coatings. (Plenum, New York, 1991)

[8] See, for example, B. S. Satyanarayana, A. Hart, W. I. Milne, and J. Robertson, Appl. Phys. Lett. 71, 1430 (1997) and references therein.

[9] H. Park, Y. K. Hong, J. S. Kim, C. Park, and J. K. Kim, Appl. Phys. Lett. 69, 779 (1996)

[10] M. Chhowalla, J. Robertson, C. W. Chen, S. R. P. Silva, C. A. Davis, G. A. J. Amaratunga, and W. I. Milne, J. Appl. Phys. 81, 139 (1997)

[11] Y. Tifshitz, G. D. Iempert, and E. Grossmann, Phys. Rev. Lett. 72, 2753 (1994)

[12] C. A. Davis, G. A. J. Amaratunga, and K. M. Knowles, Phys. Rev. Lett. 80, 3280 (1998)

[13] P. C. Kelires, J. Non-Cryst. Solids 227-230, 597 (1998)

[14] J. Dong, D. A. Drabold, Phys. Rev. B 57, 15591 (1998). 
[15] S. Uhlmann, T. Frauenheim, and Y. Lifshitz, Phys. Rev. Lett. 81, 641 (1998)

[16] C. Xu, C. Z. Wang, C. T. Chan, and K. M. Ho, J. Phys. Condensed Matter 4, 6047 (1992).

[17] M. S. Tang, C. 7. Wang, C. T. Chan, and K. M. Ho, Phys. Rev. B 53, 979 (1996).

[18] J. Kim, F. Mauri, G. Galli, Phys. Rev. B 52, 1640 (1995).

[19] R. Haerle, A. Baldereschi, and G. Galli, Appl. Phys. Lett. (in press).

[20] G. Galli, F. Gygi, and A. Catellani, Phys. Rev. Lett. 82, 3476 (1999).

[21] R. Car, M. Parrinello, Phys. Rev. Lett., 55, 2471 (1985)

[22] We used the first principles molecular dynamics code JEEP (F. Gygi, Lawrence Livermore National Laboratory, 1999). 


\section{TABLES}

TABLE I. Results for the different systems examined in our tight-binding MD simulations (see text): formation energy $\Delta E$, thickness $\Delta z$, number $N_{s}$ of atoms within a surface layer, 3- and 4-fold coordination fractions within this layer, and roughness $R_{a} .1 / \mathrm{u}$ refers to the lower and the $\underline{\underline{u}}$ pper surface respectively.

\begin{tabular}{|c|c|c|c|c|c|c|}
\hline System & $\begin{array}{c}\Delta E \\
{\left[\frac{e V}{A^{2}}\right]}\end{array}$ & $\begin{array}{l}\Delta z \\
{[\AA]}\end{array}$ & $N_{s}$ & $\begin{array}{l}N_{3} \\
{[\%]}\end{array}$ & $\begin{array}{l}N_{4} \\
{[\%]}\end{array}$ & $\begin{array}{l}R_{a} \\
[\AA]]\end{array}$ \\
\hline$\rho 2-\mathrm{TC} 1 / \mathrm{u}$ & 0.16 & $2.9 / 3.7$ & $60 / 60$ & $48 / 50$ & $3 / 0$ & $1.3 / 1.2$ \\
\hline$\rho 3-\mathrm{TC} 1 / \mathrm{u}$ & 0.17 & $3.5 / 3.5$ & $60 / 50$ & $50 / 56$ & $5 / 10$ & $0.8 / 0.8$ \\
\hline$\rho 3-\mathrm{ED} 1 / \mathrm{u}$ & 0.09 & $2.8 / 3.6$ & $35 / 40$ & $49 / 58$ & $17 / 15$ & $0.5 / 0.9$ \\
\hline$\rho 4-\mathrm{TC} 1 / \mathrm{u}$ & 0.11 & $1.4 / 2.0$ & $40 / 50$ & $70 / 62$ & $3 / 12$ & $0.4 / 0.4$ \\
\hline$\rho 4-\operatorname{ED~} 1 / \mathrm{u}$ & 0.05 & $1.9 / 1.3$ & $35 / 35$ & $71 / 65$ & $11 / 18$ & $0.3 / 0.3$ \\
\hline
\end{tabular}




\section{FIGURES}

FIG. 1. Structure of a computer generated a-C network with density $\rho=3.0 \mathrm{~g} \cdot \mathrm{cm}^{-3}(\rho 3-\mathrm{ED})$, before applying strain (a), and after the application of two strain steps with displacement $t_{2}=0.5 \AA$ prior to (b) and after (c) relaxation of the ionic coordinates. The final fully-relaxed fractured network with two surfaces is shown in (d) Black spheres represent 4-fold coordinated atoms and grey spheres represent atoms with coordination less than four.
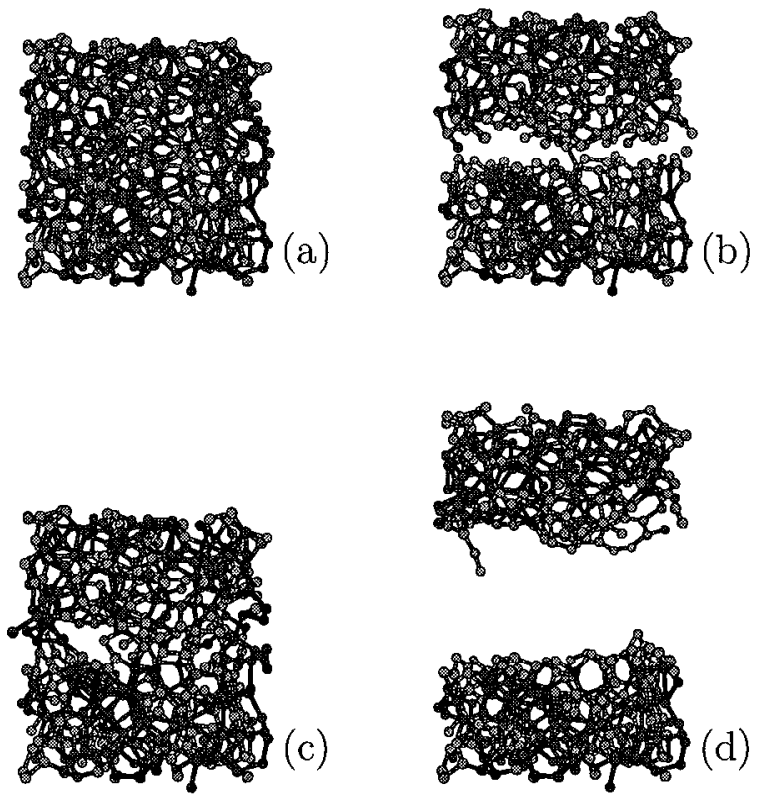

FIG. 2. Slab-averaged atomic density (A) of $n$-fold $(n=1,2,3,4)$ coordinated atoms as a function of distance from an arbitrary plane parallel to the surface for the computer generated network with bulk density $\rho=3.0 \mathrm{~g} \cdot \mathrm{cm}^{-3}(\rho 3$-ED). Density of 4-fold coordinated atoms (dotted line), 3-fold coordinated ones (broken line) and 2- plus 1-fold coordinated ones (solid line).

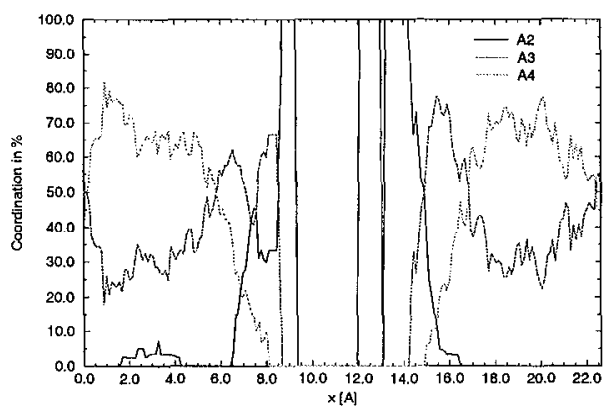

